\title{
Elastic envelopes of porous sandstones
}

\author{
Yves Guéguen ${ }^{1}$ and Jérôme Fortin ${ }^{1}$ \\ Received 23 April 2013; revised 11 June 2013; accepted 14 June 2013; published 19 July 2013.
}

[1] In this paper we focus on the case of sandstones for which many experimental data are available. We present a simple 2-D model derived from granular media mechanics. This model assumes that the granular microstructure is a key point to understand the mechanical behavior. We consider a periodic grain network and focus on the first-order neighbors of a given grain. These approximations are sufficient to explain the overall mechanical behavior in the $Q$ versus $P$ stress space. In the low pressure range, the controlling micromechanism is assumed to be tensile failure at grain contacts. The "dilatant" envelope is found to be a straight line in the stress space. In the high pressure range, the controlling micromechanism is assumed to be grain fragmentation. The "compactant" envelope is found to be a straight line in the stress space. We observed that this 2-D model slightly overestimates $Q$ versus $P$ slopes determined experimentally (2.3 instead of 1.5), which can be explained by the approximations made. Citation: Guéguen, Y., and J. Fortin (2013), Elastic envelopes of porous sandstones, Geophys. Res. Lett., 40, 3550-3555, doi:10.1002/grl.50676.

\section{Introduction}

[2] Porous rocks are present in the upper crust probably down to $5-10 \mathrm{~km}$ depth. The first $10 \mathrm{~km}$ of the earth are an ambiguous medium that is solid, but has the capability of retaining fluids. For that reason, porous rocks play a key role as they are the sites for hydrocarbon resources and for underground storage. These rocks have been investigated for many years. Their transport properties are obviously of great importance. The same is true for their elastic and, more generally, mechanical properties.

[3] A specific property of porous rocks is that they can compact. Field investigations [Mollema and Antonellini, 1996] have suggested earlier that compaction bands exist in sandstones at the field scale. The result of compaction is a drastic change of properties, in particular, of permeability. Bésuelle [2001] and Klein et al. [2001] have shown experimentally that localized failure is taking place in porous sandstones submitted to compression. They reported that compaction bands could be developed in laboratory conditions. Whether the localized features observed at the field scale are equivalent or not to the localized crushed zones obtained in laboratory experiments remains however an open question.

[4] The observed behavior indeed is theoretically expected from bifurcation theory [Rudnicki, 2002; Rudnicki

\footnotetext{
${ }^{1}$ Laboratoire de Géologie, Ecole Normale Supérieure, Paris, France.

Corresponding author: J. Fortin, Laboratoire de Géologie, Ecole Normale Supérieure, CNRS, UMR 8538, Paris, France. (fortin@geologie.ens.fr)

(C)2013. American Geophysical Union. All Rights Reserved. 0094-8276/13/10.1002/grl.50676
}

and Rice, 1975]. Experimentally, further studies have confirmed that compaction localization does occur for rocks that have a sufficiently high porosity [Baud et al., 2004, 2006; Fortin et al., 2006, Stanchits et al., 2009]. More recently, a similar behavior has been observed for the first time in basalt [Loaiza et al., 2012; Adelinet et al., 2013], showing that nonsedimentary, but porous, rocks could exhibit localized strain as well in compaction.

[5] Two common points are characteristic of the reported triaxial cell experimental investigations on sandstones. The first one is that the mechanical response is elastic within a well-defined closed domain in the (differential stress $Q$, mean effective stress $P$ ) plane. This domain is defined by a quasi-straight line of positive slope, up to a maximum pressure $P_{M}$. This is for the brittle limit. Beyond $P_{M}$, the domain is defined by a negative slope envelope that corresponds to a compacting behavior and that closes the elastic domain at $P^{*}$. The second point is that the dominant microstructural feature, when crossing the envelope, is found to be a localized (dilatant) shear band below $P_{M}$, and beyond $P_{M}$, localized (compactant) irregular crushed zones approximately perpendicular to the main compressive stress. They have been called "compaction bands".

[6] In the present paper, we show that a 2-D simple model derived from granular media mechanics accounts reasonably well for both points in the case of sandstones. The model is presented first. Its mechanical implications are compared to mechanical data next. Finally, its microstructural implications are discussed by comparison to the observed microstructures of compacted porous sandstones.

\section{2-D Granular Model}

[7] Mechanical properties of porous rocks can be analyzed from two complementary points of view. One view is to assume a continuous medium in which pores (and cracks) can be considered as inclusions. This approach has been successfully developed many years ago for elastic properties [Mackenzie, 1950; Eshelby, 1957]. The lower is the porosity, the better is the model. At the other extreme, another view is to consider a porous rock as a granular medium [Brandt, 1955]. Then, grain contacts are key features. As far as elastic properties are concerned, Mindlin [1949] results on elastic bodies in contact are then of direct interest. Real porous rocks, however, fall between these two extreme viewpoints, because they are made of grains that are cemented. Depending on the porosity value, one or the other of the two previous simplified viewpoints may be more relevant. The inclusion models are appropriate for low porosity rocks. They have been improved to take into account higher porosity values by using self-consistent models [Budiansky and O'Connell, 1975] or differential self-consistent models [Le Ravalec and Guéguen, 1996]. The granular models are more appropriate for high porosity rocks. They have been 
a)

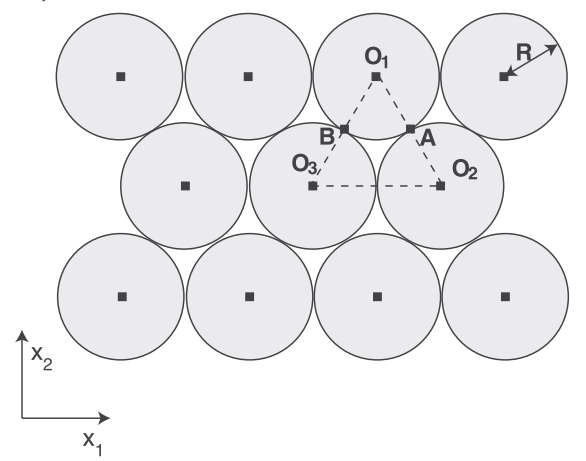

b)

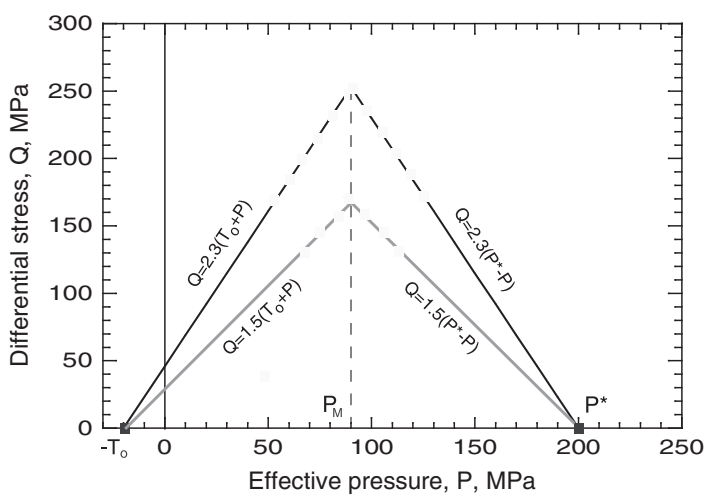

Figure 1. (a) 2-D hexagonal network of grain. (b) 2-D granular model predictions (black line); averaged experimental data (gray line) (from Figures $3 \mathrm{a}-3 \mathrm{c}$ ).

improved by bonding grains [Digby, 1981] or considering identical spherical grains and focusing on the contact of two spheres [Walton, 1960] as a key feature.

[8] Because we are interested here in the inelastic behavior (at the limit where the rock leaves the elastic domain) of porous rocks of sufficiently high porosity, the second viewpoint is the most appropriate in our case. This is specially true for sandstones that are, at least partially, granular rocks. Inelastic deformation of granular materials has been the subject of many investigations in recent years [Radjaï et al., 2004]. They have shown that the grains interact via contacts, and that this is the main point to focus on for granular materials inelastic deformation. The simplest, lowest-order model should take into account the first neighbors grains [Radjaï et al., 2004], because coordination number $z$ is a fundamental parameter. This parameter has been shown to provide more microstructural information than porosity $\Phi$, because each grain has an integer number of contacts. The coordination number is different in 2-D or 3-D models. However, 2-D models are useful because they can capture the key features. Although cohesive granular media have been less investigated, Radjaï and Richefeu [2009] have taken into account cohesion of contacts by considering local adhesion and presented a framework that can allow examining the shear strength of cohesive granular materials. The following model is inspired from these results, using a simplifying assumption: we assume here that parameter $z$ is the basic parameter to build a simplified model. The following model can be considered as well as a periodic model, equivalent to regular 3-D cubic packings ( $\mathrm{sc}$ : simple cubic, hc: hexagonal close packing, or fcc: face-centered cubic). These three periodic packings correspond to noncompact packing (sc porosity is $\Phi=0.47$, with $z=6$ ) and compact ones (hc and fcc porosity is $\Phi=0.26$, with $z=12$ ). These are useful to model porosity of sandstones [Lemée and Guéguen, 1996; Manificat and Guéguen, 1998]. More sophisticated models could of course bring more detailed results. But because this lowest-order model, as shown below, can explain-at least in several cases - the main characteristics of elastic envelopes of sandstones, we think that it is a useful one.

[9] Consider a compact structure made of identical discs of radius $R$ (Figure 1a). The 2-D model that is shown on Figure 1a corresponds to $z=6$ contact neighbors. Transposition to a 3-D model would imply $z=12$ and a porosity $\Phi=$ 0.26 , a realistic value for sandstones. As explained above, we focus on the 2-D model. Note that we consider a compact packing. The basic pattern to be considered in that case is the triangle $\mathrm{O}_{1} \mathrm{O}_{2} \mathrm{O}_{3}$ that is constructed from the centers of three neighboring grains. This granular array is isotropic for elastic properties but not for strength or failure properties. Let us consider the 2-D grain packing as a system submitted to two stress tensors: one is isotropic (pressure $P=\sigma_{11}=\sigma_{22}$ ), and the other is a shear stress $\left(Q=2 \sigma_{12}=2 \sigma_{21}\right)$. Note that the axes are chosen in such a way that the shear stress $Q$ and the segment $\mathrm{O}_{2} \mathrm{O}_{3}$ are parallel to the $x_{1}$ axis. This point will be discussed further later on. We are interested in the local pressure at point $\mathrm{A}, P_{A}$, in the middle of segment $O_{1} O_{2}$. Point A is the contact point between grains $O_{1}$ and $O_{2}$. We assume here that the macroscopic pressure $P$ is fully transmitted through the local contact plane (that is normal to $O_{1} O_{2}$ segment, i.e., at $2 \pi / 3$ of the horizontal axis). This is of course a simplification, consistent with the basic assumptions of this lowest-order model. Using the above decomposition in two tensors, we calculate $P_{A}$ as the sum of $P$ and of a second term due to the $Q$ tensor. This last tensor has two principal axes at $\pi / 4$ and $3 \pi / 4$ from the horizontal axis. Its principal stresses are $+Q / 2$ and $-Q / 2$, but they are not perpendicular (nor parallel) to the contact plane. Again, we assume that the two principal stresses $\pm Q / 2$ are fully transmitted to contact A. Noting that the angle between the contact plane and the first principal axis of the $Q$ tensor is $\pi / 12\left(15^{\circ}\right)$, we get the following:

$$
P_{A}=P+\left(\cos ^{2} \frac{\pi}{12}-\sin ^{2} \frac{\pi}{12}\right) \frac{Q}{2}=P+0.43 Q .
$$

[10] Contact A experiences a pressure $P_{A}$ higher than $P$, and $P_{A}$ increases if $Q$ increases. We assume that failure will locally take place at point $A$ when the local pressure reaches a limit $P^{*}$ (Hertzian cracks). As discussed below, this is what is evidenced from microstructural observations [Wong and Baud, 2012]. It results that, in the $P-Q$ space, a linear relation is expected:

$$
Q=2.3\left(P^{*}-P\right) \text {. }
$$

Using a similar calculation, pressure $P_{B}$ at point $\mathrm{B}$ in the middle of segment $O_{1} O_{3}$ is found to be

$$
P_{B}=P-\left(\cos ^{2} \frac{\pi}{12}-\sin ^{2} \frac{\pi}{12}\right) \frac{Q}{2}=P-0.43 Q .
$$


[11] Contact B experiences a pressure $P_{B}$ lower than $P$, and $P_{B}$ decreases if $Q$ increases. We assume that failure will locally take place at point $B$ when the local pressure reaches a zero value, or maybe a small negative value (strength in traction $\left.-T_{0}\right)$. It results that, in the $P-Q$ space, a linear relation is expected:

$$
Q=2.3\left(T_{0}+P\right)
$$

The previous results are equivalently obtained in calculating directly the normal component of the stress vector $n_{i} \sigma_{i j} n_{j}$ on the tangent planes in $\mathrm{A}$ and $\mathrm{B}$.

[12] The model predicts an elastic domain limited by two straight lines of slope \pm 2.3 in the $P-Q$ space. The maximum $Q$ value is found to be $Q_{M}=1.15\left(P^{*}+T_{0}\right)$ and $P_{M}=\frac{P^{*}-T_{0}}{2}$.

[13] The overall result is shown in Figure $1 \mathrm{~b}$ in the $P-Q$ space, for arbitrary values of $P^{*}=200 \mathrm{MPa}$ and $T_{0}=20$ $\mathrm{MPa}$. In a $2-\mathrm{D}$ model, $P=\frac{\sigma_{1}+\sigma_{2}}{2}$ and $Q=\left(\sigma_{1}-\sigma_{2}\right)$ where $\sigma_{1}$ is the maximum principal stress (using the convention that compressive stresses are taken positive) and $\sigma_{2}$ is the minimum compressive stress. Alternatively, in the principal stresses plane, $\sigma_{1}$, and $\sigma_{2}$ plane, the elastic domain can be defined from the above results by the two straight lines corresponding respectively to the compaction and dilatant limits. The fact that linear boundaries are predicted is not surprising, since the considered micromechanical processes are linear.

[14] We pointed earlier that the $x_{1}$ axis (Figure 1a) is parallel to segment $\mathrm{O}_{2} \mathrm{O}_{3}$. In the general case, it could be at any angle from $\mathrm{O}_{2} \mathrm{O}_{3}$. Let us consider a rotation of the array such as the angle $\left[\mathrm{O}_{2} \mathrm{O}_{3} ; x_{1}\right.$ axis $]=\theta$. Then, instead of $(\pi / 12)$ in the previous calculations, the angle should be $(\pi / 12+\theta)$. It follows that, instead of the expression $\left[\cos ^{2}(\pi / 12)-\sin ^{2}(\pi / 12)\right]=\cos (\pi / 6)$, we have $\cos (\pi / 6+2 \theta)$. Varying $\theta$ from 0 to $\pi / 6$ is sufficient because of the hexagonal symmetry. The result is that the predicted slopes in the $(Q, P)$ plot are larger than 2.3 , which is the minimum possible value. Given that any real rock is not a regular array of grains, $\theta$ should be considered as variable from one place to the other. However, the lowest slope value is reached for $\theta=0$, and this should be the place where failure starts.

\section{Local Failure Mechanisms}

[15] The local failure mechanisms are different at points $\mathrm{A}$ and $\mathrm{B}$, although in both cases, tensile failure is involved. Consider first point A. Zhang et al. [1990] have analyzed the failure process at $Q=0, P=P^{*}$, using the [Brandt, 1955] model to relate the local force at grain contact $F$ to pressure $P$. Assuming that the local force $F$ reaches a critical value $F_{c}$, corresponding to the critical value of the stress intensity factor $K_{I}$, they have derived a relation between $P^{*}$, grain size $R$ and porosity. At $P^{*}$, grain fragmentation takes place. Our model extends their analysis to pressure values lower than $P^{*}$. The basic mechanism at grain contact $\mathrm{A}$ is assumed to be the same for all pressures in the interval $\left[P_{M}, P^{*}\right]$. To be consistent with the above granular medium description, it is more appropriate, however, to use the Walton [1960] model to relate $F$ and $P$. The Walton model assumes a random packing of identical spheres whereas the Brandt model assumes a kind of fractal packing. The Walton model is thus closer to the real situation of sandstone than the Brandt model, and this is an additional reason to use it. It predicts that $F=4 \pi R^{2} P / z(1-\Phi)$, where $z$ is the number of contacts/grain. Assuming $z=6$, the critical $P^{*}$ value is reached for a critical force $F_{c}=2 \pi R^{2} P^{*} / 3(1-\Phi)$. We follow Zhang et al. [1990] and assume that local failure is reached when the stress intensity factor $K_{I}=1.12 \sigma_{r}(\pi c)^{-1 / 2}=K_{I c}$ for a microcrack of length $c$, with a tensile stress $\sigma_{r}$ at the crack tip. The $\sigma_{r}$ value is $\sigma_{r}=(1-2 \nu) F / 2 \pi a^{2}$ where $a$ is the Hertz contact radius. Then

$$
P^{*}=16\left[\left(1-v^{2}\right) / E\right]^{2}\left[K_{I c} /(1-2 v)\right]^{3}(1-\Phi) c^{-3 / 2},
$$

where $E$ is the Young modulus and $v$ the Poisson ratio of the grains. Note that the expression for $F_{c}$ from Walton model yields a finite value for $P^{*}$ at the limit of vanishingly small porosity, whereas that of Zhang et al. [1990] diverges because it varies as $\Phi^{-3 / 2}$. The above equation, however, predicts an increase of $P^{*}$ if porosity decreases, as Zhang et al. [1990] model. Moreover, if $c$ scales as the grain size $R$ as assumed by Zhang et al. [1990], the grain size dependence is found to be $R^{-3 / 2}$. Because $\left(K_{I c}\right)^{2}=G_{I c} E$, where $G_{I c}$ is the critical strain release rate, the above expression can be modified. Assuming a Poisson ratio of 0.1 , an average value realistic for quartz (this parameter is not a key one for the exact $P^{*}$ value), the previous result is changed into

$$
P^{*}=30(E)^{-1 / 2}\left(G_{I c}\right)^{3 / 2}(1-\Phi) c^{-3 / 2} .
$$

If we assume a $P^{*}$ value of $200 \mathrm{MPa}$, a porosity of 0.2 and a Young's modulus of $70 \mathrm{GPa}$, then $c=0.13 \mu \mathrm{m}$ if $G_{I c}=100$ $\mathrm{J} \mathrm{m}^{-2}$. Crack length value varies linearly with $G_{I c}$ value if all other parameters are constant. This small $c$ value is in agreement with the fact that local Hertz cracks are expected to develop at a very small scale, smaller than that of contact radius $a$. Therefore, $c$ should be much smaller than the grain size. Large $c$ values would imply large $G_{I c}$ values that are not realistic. Smaller $G_{I c}$ values $\left(10 \mathrm{~J} \mathrm{~m}^{-2}\right)$ would imply smaller crack lengths $(0.01 \mu \mathrm{m}) . P^{*}$ values smaller than $200 \mathrm{MPa}$ could correspond to either lower $G_{I c}$ values or larger crack length compared to the above values. Figure 2 shows a compilation of published data [Wong et al., 1997; Baud et al., 2006; Fortin et al., 2007; Wong and Baud, 2012; Rutter and Glover, 2012] plotted in a plane $\left(P^{*},(1-\Phi) R^{-3 / 2}\right)$ assuming that $c$ is proportional to $R$ and taking into account the $(1-\Phi)$ factor.

[16] Let us now consider point B. The local mechanism is completely different. In the lower pressure range $\left[0, P_{M}\right]$, we assume local failure at $\mathrm{B}$ when $P_{B}$ is zero, or more likely slightly negative if the grain contact has a local tensile strength $\left(-T_{0}\right)$. The overall failure is a shear band, but the local failure is a tensile one. Note that the $T_{0}$ value is likely to depend also on porosity and grain size. An estimate of $T_{0}$ can be found by using a similar method to that used in the previous case for estimating $P^{*}$. Tensile failure at grain boundary takes place when the tensile local force at grain boundary reaches a critical value such as $K_{I}=K_{I c}$, for a microcrack of length $c$. An important difference with the previous case is that the local force is applied over a grain boundary (cemented) surface $\pi r^{2}$ and not over the Hertzian contact area $\pi a^{2}$. One expects $r \gg a$. Indeed, the $r$ value should be smaller but close to the grain radius $R$. For simplicity, we assume $r=R / 2$. Taking into account these modifications, one gets the following:

$$
T_{0} \simeq 0.5\left[G_{I c} E\right]^{1 / 2}(1-\Phi) c^{-1 / 2}
$$




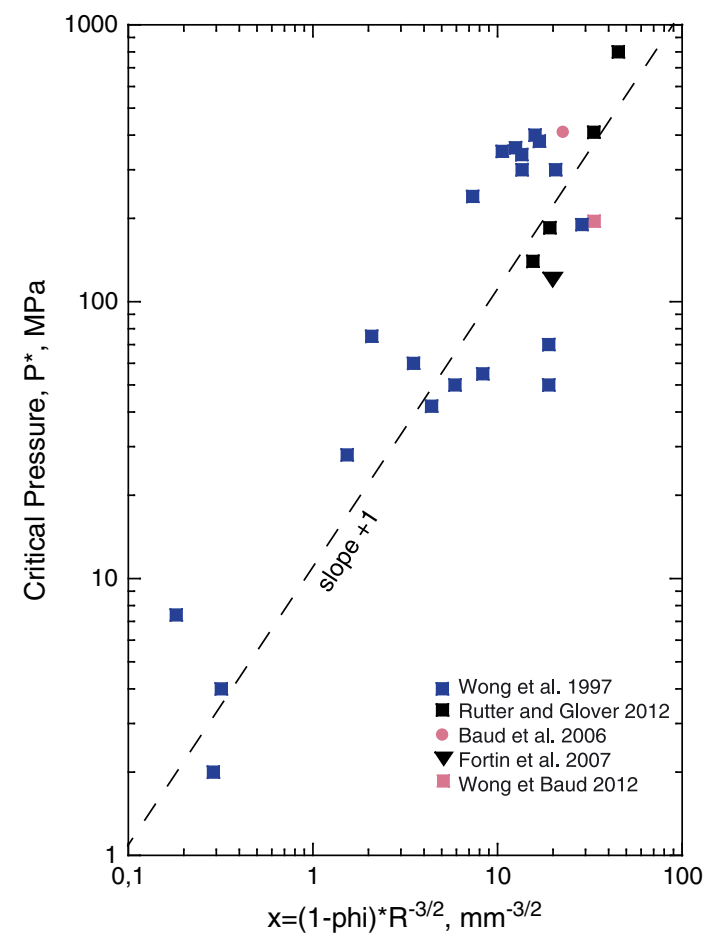

Figure 2. Critical pressure $P^{*}$ as a function of $x=$ $(1-\Phi) R^{-3 / 2}$, where $x$ is in $\left(\mathrm{mm}^{-3 / 2}\right)$. Each data point corresponds to a given rock of given values $(\Phi, R)$. The model predicts a slope +1 . The general trend is consistent with the experimental data, with some scatter.

If, as above, we assume $\Phi=0.2, E=70 \mathrm{GPa}, G_{I c}=10$ $\mathrm{J} \mathrm{m}^{-2}$, one gets $T_{0} \approx 60 \mathrm{MPa}$ for $c=10 \mu \mathrm{m}$. The $c$ value should be larger than in the Hertzian crack case because the small Hertzian contact radius $a$ has been changed into the larger $r$ grain boundary radius. Thus, larger crack sizes should be considered.

\section{Elastic Envelope: Predicted and Observed Results}

[17] Published data on sandstone compaction have shown that the elastic envelope in the $P-Q$ space is built of two parts. The first part-low $\mathrm{P}$, dilatant regime - is a linear one, consistent with the above result. This is the well-known Coulomb straight line. As noted by Rutter and Glover [2012], the friction slope is 1.43 for axisymmetric loading. Comparison of the 2-D model to the experimental results is not straightforward, however, because experimental results are 3-D axisymmetric loading results. According to Figure 3, the observed slopes are close to 1.5 (with an error of \pm 0.1 ). This is smaller than the above predicted 2-D value (2.3). Yet it is likely that the 2-D granular model overestimates the slope value for a reason directly linked to the approximations made. The main assumption is that the rock is considered to be a granular packing, with point contacts between grains. This seems to be a sufficiently good assumption to derive the overall shape of the elastic envelope. It does not allow us, however, to calculate the exact slope values. We suggest that this is because cementation is not taken into account. Contacts between grains are not points, like $\mathrm{A}$ and $\mathrm{B}$, but thin cemented zones. Such zones have a nonzero thickness and the stress vector projection on the contact tangent plane plays a role that has been ignored so far. A straightforward calculation shows that this effect results in decreasing the effect of $P$, and increasing the effect of $Q$. The consequence is that the $Q$ versus $P$ slope has been overestimated. Given that (in the above model) grain packing is a compact one, it is not expected that the breakdown of cementation will tend to cause a more compact arrangement. Overall dilation is expected.

[18] The second part of the elastic envelope (high $P$ ) is compactive, because grain fragmentation causes a decrease of porosity and grain size according to the model. The compactant boundary is in general considered to be closer to an elliptical shape [Wong and Baud, 2012], although a straight line of negative slope has also been reported [Fortin et al., 2006, 2009]. The exact slope is experimentally determined with a limited accuracy. The question is: Up to what extent does the high $P$ experimental envelope differ from a straight line? Figure 3 shows several examples for different sandstones. Depending on the precise rock, a straight line is more or less well evidenced.

[19] Let us first consider the $P-Q$ domain close to $\left[P_{M}, Q_{M}\right]$. In this domain, where the dominant behavior shifts from a dilatant regime to a compactant regime, local grain contacts shift from decohesion (B contacts) to Hertz cracks (A contacts). Our lowest-order model cannot capture the detailed behavior in this intermediate regime where fluctuations of coordination number $z$, of angular orientations of contacts (hence of local forces), make the predictions difficult. Because the overall effects of $P$ and $Q$ are almost canceling each other, small packing variations become very important. A regular periodic packing cannot account for the exact behavior then. Indeed, because random fluctuations play a key role in that case, it is expected that experimental data could vary from one sample to the other (for the same rock). We suggest thus, for fundamental reasons, that it is not possible to rely strongly on the results obtained near $Q_{M}$ (unless a large set of data is obtained on similar samples to get a statistically significant result). In addition, a more sophisticated model, following Radjaï et al. [2004], would be required.

[20] Let us next consider the $P-Q$ domain at high $P$ values. It seems possible to fit the data points in the high $P$ regime with a straight line at least for Figures $3 a-3 c$. Its slope varies around 1.5. It is smaller than the slope predicted by the 2-D model. Again accounting for a cementation shows that the 2-D granular model overestimates the slope value. Is a straight line more appropriate than an elliptical cap model to fit the data? Looking at Figure 3, it is an open question. However, an elliptical cap (the main axis of which is the $P$ axis) would imply a vertical tangent close to the $P$ axis, at $P=P^{*}$. This would imply that the behavior is no longer $Q$-dependent near $P^{*}$. But there are, however, no data near the $Q=0$ axis. In any case, the model can be an associated one [Rudnicki, 2004] although Radjaï [2009] pointed out that granular plasticity models are in general nonassociated models.

\section{Compaction Bands: Cascading Failure}

[21] A striking feature of samples deformed in the compaction regime is that they show "compaction bands" that 

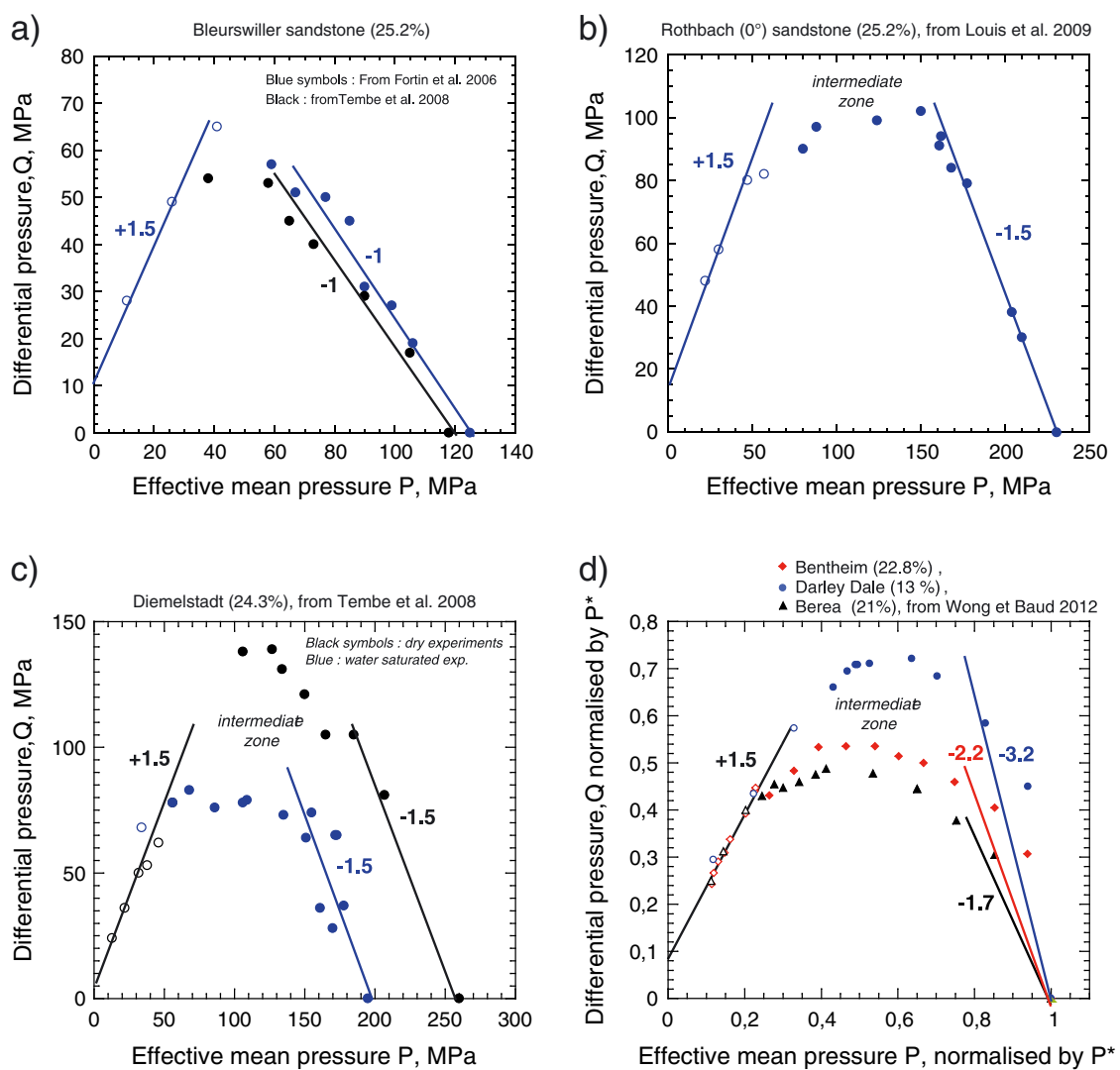

Figure 3. (a) Data for Bleurswiller sandstone (blue dots: data obtained by Fortin et al., [2006, 2009], black dots: data obtained by Tembe et al. [2008]). The envelope is well described by two straight lines of slopes $+1.5 \pm 0.1$ and $-1 \pm 0.1$. (b) Data for Rothbach sandstone from Louis et al. [2009]. The envelope is well-described by two straight lines of slopes $\pm 1.5 \pm 0.1$ and an intermediate zone. (c) Data for Diemelstadt sandstone from Tembe et al. [2008]. The envelope is welldescribed by two straight lines of slopes $\pm 1.5 \pm 0.1$ and an intermediate zone. (d) Normalized data for Bentheim, Darley Dale, and Berea sandstones from Wong and Baud [2012]. For these sandstones, the slope and shape of the compactant envelopes are variable.

are narrow layers, approximately one grain size thick, approximately horizontal, but irregular. These layers are formed of crushed grains and collapsed pores and are about two or three grains thick for homogeneous sandstones [Wong and Baud, 2012]. This observation has been used as supporting the Hertzian crack model. Although the layers are approximately perpendicular to the main compressive stress, they are made of irregular segments that are interpreted as segments $O_{1} O_{2}$ of Figure 1. This implies that once local failure has taken place at point $\mathrm{A}$, the local high normal stress is transferred to the neighboring unfailed grain contacts. Moreover, Hertzian cracks are formed on either side of $\mathrm{A}$ and are approximately normal to the A contact plane. These cracks build up an increased local compressive stress near B, on B contact planes. It is so because parallel microcracks add up their effects and thus amplify the local normal stress on the contact plane at B [Orlowsky et al., 2003]. It results that a first local failure at A point induces a second local failure at point $\mathrm{B}$. The overall expected pattern is a zigzagging band as the observed one (see, for example, Schultz et al. [2010, Figure 1c], where they report field observations). A cascading failure is produced, roughly horizontal, but following a zigzag path.

\section{Conclusion}

[22] Granular mechanics is potentially very useful to investigate porous rocks mechanical behavior. The granular microstructure is described by the network of grain contacts, so that the coordination number $z$ is a key parameter. Considering porous sandstones as a granular medium, and using a 2-D model where grains are packed according to a $z=6$ compact packing, the two main characteristics of the inelastic behavior of sandstones are derived: (1) a lowpressure elastic limit that is a straight line in the $P-Q$ plane, corresponding to a dilatant shear band controlled by grain boundary tensile failure, (2) a high pressure elastic limit that is also a straight line in the same plane, corresponding to compaction bands and controlled by grain fragmentation. Up to what point other porous rocks (for instance limestones, basalts) could be investigated along similar views remain to be explored. An improvement of the model would be to quantitatively account for the cement at grain contacts.

[23] Acknowledgments. The authors thank John Rudnicki and an anonymous reviewer for their helpful comments.

[24] The Editor thanks two anonymous reviewers for their assistance in evaluating this paper. 


\section{GUEGUEN AND FORTIN: ELASTIC ENVELOPES OF POROUS SANDSTONES}

\section{References}

Adelinet, M., J. Fortin, A. Schubnel, and Y. Guéguen (2013), Deformation modes in an Icelandic basalt: From brittle failure to localized deformation bands, J. Volcanol. Geotherm. Res., 255, 15-25, doi:10.1016/ j.jvolgeores.2013.01.011.

Baud, P., E. Klein, and T. Wong (2004), Compaction localization in porous sandstones: Spatial evolution of damage and acoustic emission activity, J. Struct. Geol., 26, 603-624, doi:10.1016/j.jsg.2003. 09.002 .

Baud, P., V. Vajdova, and T. Wong (2006), Shear-enhanced compaction and strain localization: Mechanical data and constitutive parameters for porous sandstones, J. Geophys. Res., 111, B12401, doi:10.1029/ 2005JB004101.

Bésuelle, P. (2001), Compacting and dilating shear bands in porous rocks: Theoretical and experimental conditions, J. Geophys. Res., 106(B7), 13,435-13,442, doi:10.1029/2001JB900011.

Brandt, H. (1955), A study of the speed of sound in porous granular media, J. Appl. Mech., 22, 479-486.

Budiansky, B., and R. J. O'Connell (1975), Elastic moduli of dry and saturated cracked solids, Int. J. Solids Struct., 12, 81-97.

Digby, P. J. (1981), The effective elastic moduli of porous granular rocks, J. Appl. Mech., 48, 803-808.

Eshelby, J. D. (1957), The determination of the elastic field of an ellipsoidal inclusion, and related problems, Proc. R. Soc. London, Ser. A, 241, 376-396.

Fortin, J., S. Stanchits, G. Dresen, and Y. Guéguen (2006), Acoustic emission and velocities associated with the formation of compaction bands in sandstone, J. Geophys. Res., 111, B10203, doi:10.1029/2005JB003854.

Fortin, J., A. Schubnel, and Y. Guéguen (2007), Effect of pore collapse and grain crushing on ultrasonic velocities and Vp/Vs, J. Geophys. Res., 112, B08207, doi:10.129/2005JB004005.

Fortin, J., S. Stanchits, G. Dresen, and Y. Guéguen (2009), Acoustic emissions monitoring during inelastic deformation of porous sandstone: Comparison of three modes of deformation, Pure Appl. Geophys., 166, 823-841, doi:10.1007/s00024-009-0479-0.

Klein, E., P. Baud, T. Reuschlé, and T. Wong (2001), Mechanical behaviour and failure mode of Bentheim sandstone under triaxial compression, Phys. Chem. Earth, 26, (21-25).

Lemée, C., and Y. Guéguen (1996), Modeling of porosity loss during compaction and cementation of sandstones, Geology, 24(10), $875-878$.

Le Ravalec, M., and Y. Guéguen (1996), High and low frequency elastic moduli for a saturated porous/cracked rock, Geophysics, 61, 1080-1094.

Loaiza, S., J. Fortin, A. Schubnel, Y. Guéguen, S. Vinciguerra, and M. Moreira (2012), Mechanical behavior and localized failure modes in a porous basalt from the Azores, Geophys. Res. Lett., 39, L19304, doi:10.129/2012GL053218.

Louis, L, P. Baud, and T.-F. Wong (2009), Microstructural inhomogeneity and mechanical anisotropy associated with bedding in sandstone, Pure Appl. Geophys., 166, 1063-1087, doi:10.1007/s00024-009-0486-1.
Mackenzie, J. K. (1950), The elastic constants of a solid containing spherical holes, J. Geophys. Res., B63(2), 2-11.

Manificat, G., and Y. Guéguen (1998), What does control Vp/Vs in granular rocks? Geophys. Res. Lett., 25, 381-384.

Mindlin, R. D. (1949), Compliance of elastic bodies in contact, J. Appl. Mech., 16, 259-268.

Mollema, P. N., and M. A. Antonellini (1996), Compaction bands: A structural analogue for anti-mode I cracks in Aeolian sandstone, Tectonophysics, 167, 209-228.

Orlowsky, B., E. H. Saenger, Y. Guéguen, and S. Shapiro (2003), Effects of parallel crack distributions on effective elastic properties-A numerical study, Int. J. Fract., 124(3-4), L171-L178.

Radjaï, F., H. Troadec, and S. Roux (2004), Key features of granular plasticity, in Granular Materials: Fundamentals and Applications, pp. 157-184, R. Soc. Chem., Cambridge, U. K. doi:10.1039/ 9781847550996-00157.

Radjaï, F., and V. Richefeu (2009), Bond anisotropy and cohesion of wet granular materials, Philos. Trans. R. Soc. A, 367, 5123-5138, doi:10.1098/rsta.2009.0185.

Radjaï, F. (2009), Force and fabric states in granular media, in Powders and Grains 2009, edited by N. Masami, and S. Luding, 35-42, Am. Inst. of Phys., New York.

Rudnicki, J. W. (2002), Conditions for compaction and shear bands in a transversely isotropic material, Int. J. Solids Struct., 39(13-14), 3741-3756

Rudnicki, J. W. (2004), Shear and compaction band formation on an elliptic yield cap, J. Geophys. Res., 109, B03402, doi:10.1029/2003JB002633.

Rudnicki, J. W., and J. R. Rice (1975), Conditions for the localization of deformation in pressure sensitive dilatant materials, J. Mech. Phys. Solids, 23, 371-394.

Rutter, E. H., and C. T. Glover (2012), The deformation of porous sandstones; Are Byerlee friction and the critical state line equivalent? J. Struct. Geol., 44, 129-140, doi:10.1016/j.jsg.2012.08. 014.

Schultz, R. A., C. H. Okubo, and H. Fossen (2010), Porosity and grain size controls on compaction band formation in Jurassic Navajo Sandstone, Geophys. Res. Lett., 37, L22306, doi:10.1029/2010GL044909.

Stanchits, S., J. Fortin, Y. Guéguen, and G. Dresen (2009), Initiation and propagation of compaction bands in dry and wet Bentheim sandstone, Pure Appl. Geophys., 166, 843-868, doi:10.1007/s00024-009-0478-1.

Tembe, S., P. Baud, and T.-F Wong (2008), Stress conditions for the propagation of discrete compaction bands in porous sandstone, J. Geophys. Res., 113(B09409), doi:org/10.1029/2007JB005439.

Walton, K. (1960), The effective elastic moduli of a random packing of spheres, J. Mech. Phys. Solids, 35(2), 213-226.

Wong, T.-F, C. David, and W. Zhu (1997), The transition from brittle faulting to cataclastic flow in porous sandstones: Mechanical deformation, J. Geophys. Res., 102, 3009-3025.

Wong, T., and P. Baud (2012), The brittle-ductile transition in porous rock: A review, J. Struct. Geol., 24, 25-53, doi:org/10.1016/j.jsg.2012.07.010.

Zhang, J., T. Wong, and D. M. Davis (1990), Micromechanics of pressureinduced grain crushing in porous rocks, J. Geophys. Res., 95, 341-352. 